\title{
Characterization of a protein-protein interaction network of the CBL-interacting protein kinase 8 from sugarcane
}

\author{
T.F. Farani, A. Gentile, R.G. Tavares, C. Ribeiro and M. Menossi \\ Laboratório de Genoma Funcional, \\ Departamento de Genética, Evolução e Bioagentes, Instituto de Biologia, \\ Universidade Estadual de Campinas, Campinas, SP, Brasil \\ Corresponding author: M. Menossi \\ E-mail: menossi@lgf.ib.unicamp.br \\ Genet. Mol. Res. 14 (1): 483-491 (2015) \\ Received July 31, 2013 \\ Accepted October 10, 2014 \\ Published January 26, 2015 \\ DOI http://dx.doi.org/10.4238/2015.January.26.1
}

\begin{abstract}
Plants are sessile organisms and have evolved to tolerate a constantly changing environment. After the onset of different stress conditions, calcineurin B-like (CBL) proteins can sense calcium signals and activate CBL-interacting protein kinase (CIPK) proteins, which can phosphorylate downstream proteins to reestablish plant homeostasis. Previous studies in the bioenergy crop sugarcane showed that the $S c C I P K 8$ gene is induced by drought stress and is also related to sucrose content. Here, we have characterized the protein-protein interactions of ScCIPK8 with six CBL proteins (ScCBL1, ScCBL2, ScCBL3, ScCBL6, ScCBL9, and ScCBL10). Yeast two-hybrid assays showed that ScCIPK8 interacts with ScCBL1, ScCBL3, and ScCBL6. Bimolecular fluorescence complementation assays confirmed in planta the interactions that were observed in yeast cells. These findings give insights on the regulatory networks related to sugar accumulation and drought stress responses in sugarcane.
\end{abstract}

Key words: ScCIPK8; Sugarcane calcineurin B-like (CBL) proteins; Yeast two-hybrid assay; Bimolecular fluorescence complementation; Calcium signaling 


\section{INTRODUCTION}

Plants have evolved to tolerate a constantly changing environment and have developed a complex signaling network to perceive and adapt to a wide range of stress conditions. Evidence suggests that for each stress condition, specific temporal, spatial, and concentration variations in cytosolic free calcium $\left(\mathrm{Ca}^{2+}\right)$ are generated (Webb et al., 1996). These calcium signatures can be decoded and modulated by calcium-binding proteins, which bind calcium through their EF-hands and relay the signal by activating kinases, transcription factor, or directly acting at promoters of effector genes (DeFalco et al., 2010; Hassan et al., 2012). One group of plant-specific calcium-binding proteins comprises the calcineurin B-like (CBL) proteins (Shi et al., 1999). CBLs have four EF-hands by which they can bind calcium and then activate a group of Ser/Thr protein kinases designated as CBL-interacting protein kinases (CIPKs) (Kudla et al., 1999). These kinases are known to be composed of an N-terminal catalytic kinase domain and a C-terminal regulatory domain, with a junction domain connecting them. A conserved activation loop, localized between the DFG and APA amino acid residues at the kinase domain, is present in all known CIPKs. It has been shown that a 24-amino acid region named the NAF/FISL motif that is localized in the regulatory domain is responsible for the CIPK/CBL interactions (Albrecht et al., 2001).

Genomic analyses revealed the existence of at least 10 CBLs and 25 CIPKs in the Arabidopsis thaliana genome and 10 CBLs and 30 CIPKs in the rice genome (Guo et al., 2001; Luan et al., 2002; Kolukisaoglu et al., 2004). CIPKs and CBLs are usually involved in abscisic acid signaling; drought, salinity, and cold responses; auxin transport; and sugar signaling (Guo et al., 2001, 2002; Gong et al., 2002; Lee et al., 2005; Xiang et al., 2007; Tripathi et al., 2009). Although these processes can initially trigger similar cellular responses like calcium influx into the cell, functional specificity can be achieved by the great number of combinatorial possibilities of CIPK/CBL networks; by their biochemical and sub-cellular localization; and by their specific spatial, temporal, and environmental gene expression patterns.

Although some CIPK/CBL pathways have been described and functionally studied in Arabidopsis thaliana, little is known about the pathways in sugarcane. Here, we describe the cloning and characterization of the ScCIPK8,ScCBL1, ScCBL2, ScCBL3, ScCBL6, $S c C B L 9$, and $S c C B L 10$ coding sequences. We also show by yeast two-hybrid and bimolecular fluorescence complementation (BiFC) assays that the ScCIPK8 protein can interact with ScCBL1, ScCBL3, and ScCBL6 but not with ScCBL2, ScCBL9, and ScCBL10 proteins.

\section{MATERIAL AND METHODS}

\section{Cloning of full-length $S c C I P K 8$ cDNA}

The sugarcane assembled sequence (SAS) SCEQLB2019B08.g from the SucestFun database (http://www.sucest-fun.org/) was used as the template to clone the ScCIPK8 open reading frame (ORF) into $p G E M$-T-Easy (Promega, USA). Total RNA from mature sugarcane leaves was used to synthesize first-strand cDNA using an oligo $(\mathrm{dT})_{20}$ primer with a Superscript III RT kit following manufacturer instructions (Invitrogen, USA). The complete coding sequence of ScCIPK8 was amplified using this first-strand cDNA 
as template with specific primers (5'-GAATTCATGAGTACAACCAAGGTGAA-3' and 5'-GGATCCACGAGGTTCTGCTAAGCAG-3') flanked by EcoRI and BamHI restriction enzyme sites.

\section{Cloning of full-length $S c C B L$ cDNAs}

ScCBL ORFs were identified after surveying the Sucest-Fun database. Ten Arabidopsis $C B L$ genes and 10 rice $C B L$ genes were used as query for the Basic Local Alignment Search Tool Algorithm. Six SASs corresponding to putative $S c C B L$ genes were identified and named according to their similarities with Arabidopsis and rice $C B L$ genes: $S c C B L 1, S c C B L 2$, $S c C B L 3, S c C B L 6, S c C B L 9$, and $S c C B L 10$ (Table 1). Specific primers flanked by restriction enzyme sites were designed to amplify the complete coding sequence of each $S c C B L: S c C B L 1$ (5'-CCCGGGGGGCGATGGGGTGCTTCCA-3': SmaI and 5'-GGATCCTCGGGGAGAGAA TCATGGTCACGT-3': BamHI), ScCBL2 (5'-GAATTCCATATCATGGTGCAGTGC-3': EcoRI and 5'-GGATCCTCAGTTATCATCGACTTGAGA-3': BamHI), ScCBL3 (5'-CATATGATGTT GCAGTGCCTGGAT-3': NdeI and 5'-CTCGAGCAGAAGTTCAGGTATCATCGAC-3': XhoI), ScCBL6 (5'-CATATGATGGTGGACTTTGTTCG-3': NdeI and 5'-CCCGGGTCACGCATC CTCTACTTG-3': SmaI), ScCBL9 (5'-GAATTCCGAATGCCACGAGCCAC-3': EcoRI and 5'-GGATCCTTAATCTTCCACTGCCGTGTTG-3': BamHI), ScCBL10 (5'-GGATCCCCAT GGACTCCTGCCGCTG-3': BamHI and 5'-CTCGAGATCGCCCTAGTCTTCGACTTG-3': $X h o I)$. The plasmid clones containing the $S c C B L$ SASs were used as template to amplify each $S c C B L$ ORF, which was then cloned into the $p G E M-T-E a s y$ vector (Promega).

Polymerase chain reaction (PCR) conditions included: $1 \mathrm{X}$ High Fidelity PCR Buffer with $\mathrm{MgCl}_{2}$ (Fermentas, USA), $0.2 \mathrm{mM}$ dNTPs mix, $0.2 \mu \mathrm{M}$ of each primer, 10-1000 ng template DNA, 1.2\% dimethyl sulfoxide, and 3 U High Fidelity PCR Enzyme Mix (Fermentas). Thermal cycling conditions included the following: an initial cycle at $94^{\circ} \mathrm{C}$ for $3 \mathrm{~min} ; 30$ cycles of $94^{\circ} \mathrm{C}$ for $30 \mathrm{~s}, 60^{\circ} \mathrm{C}$ for $30 \mathrm{~s}, 72^{\circ} \mathrm{C}$ for $2 \mathrm{~min}$; and a final extension of $72^{\circ} \mathrm{C}$ for $7 \mathrm{~min}$.

\section{Sequence alignment and domain identification}

The theoretical molecular mass and isoelectric point of the deduced amino acid sequences of ScCIPK8 and ScCBL proteins were calculated using ExPASy ProtParam Tool (Gasteiger et al., 2005). Multiple alignments with several ScCIPK proteins from diverse plants were conducted using the ClustalW2 algorithm (Larkin et al., 2007; Goujon et al., 2010). Myristoylation and palmitoylation site prediction were performed using the ExPASy Myristoylator Tool (Bologna et al., 2004) and the CSS-Palm algorithm (Ren et al., 2008).

\section{Yeast two-hybrid assays}

Yeast two-hybrid assays were conducted using the BD Matchmaker ${ }^{\mathrm{TM}}$ Library Construction and Screening Kit (Clontech, USA) following manufacturer instructions. The Sc$C I P K 8$ coding sequence was digested from the $p G E M-T$-Easy vector, purified, and cloned into the $p G B K T 7$ vector to generate the bait construct $p G B K T 7:: S c C I P K 8$. ScCBL coding sequences were digested from $p G E M-T$-Easy, purified, and cloned into the $p G A D T 7$ vector, generating the corresponding $p G A D T 7:: S c C B L$ prey constructs. Each prey construct was 
individually co-transformed with the bait construct into Saccharomyces cerevisiae AH109 cells. Control constructs given by the BD Matchmaker ${ }^{\mathrm{TM}}$ Library Construction and Screening Kit included the murine $p 53$ gene cloned into the $p G B K T 7$ vector ( $p G B K T 7:: 53$ ) as the bait construct and the human lamin $C$ gene cloned into the $p G A D T 7$ vector ( $p$ GADT7::Lam) and the SV40 Large T-antigen gene cloned into the $p G A D T 7$ vector ( $p G A D T 7:: T)$ as prey constructs. The negative control consisted of yeast AH109 cells that were co-transformed with $p$ GBKT7::53 and $p G A D T 7::$ Lam, while the positive control consisted of yeast AH109 cells that were co-transformed with $p G B K T 7:: 53$ and $p G A D T 7:: T$. Transformed yeast cells were first streaked onto selective synthetic defined medium (SD) without Trp and Leu amino acids and grown for 4 days at $30^{\circ} \mathrm{C}$. Positive colonies were then streaked onto SD medium without Trp, Leu, and His amino acids and containing $2.5 \mathrm{mM} \mathrm{3-amino-1,2,4-triazole,} \mathrm{and}$ they were grown for 4 days at $30^{\circ} \mathrm{C}$. Finally, positive colonies were streaked onto SD medium without Trp, Leu, His, and Ade and containing $0.8 \mathrm{mg} 5$-bromo-4-chloro-3-indolyl- $\alpha$ $\mathrm{D}$-galactopyranoside $(\mathrm{X}-\alpha-\mathrm{Gal})$ and grown for 4 days at $30^{\circ} \mathrm{C}$. After 4 days of incubation, positive blue colonies were selected and re-streaked on SD medium without Trp, Leu, His, and Ade and containing $0.8 \mathrm{mg} \mathrm{X}-\alpha-\mathrm{Gal}$ and grown for 4 days at $30^{\circ} \mathrm{C}$ to ensure the positive phenotype.

\section{BiFC assays}

The complete coding sequences of $S c C I P K 8, S c C B L 1, S c C B L 3$, and $S c C B L 6$ were first PCR-amplified to allow their cloning without the stop codons into the $p G E M-T$-Easy vector. The primers used and the restriction enzyme sites added were: ScCIPK8 (5'-TCTAG AATGAGTACAACCAAGGTGAA-3': XbaI and 5'-GGTACCAGCAGCATATTGCGTTT GAAGA-3': KpnI), ScCBL1 (5'-TCTAGAGCGGCGATGGGGTGCTTCC-3': $X b a \mathrm{I}$ and 5'-GGTACCCGTGACGAGATCATCGACTTCGG-3': KpnI), ScCBL3 (5'-TCTAGACGTC GATGCTTGGTTAGCAG-3': $X b a \mathrm{I}$ and 5'-CTCGAGGGTATCATCGACCTGAGAATG-3': $K p n I$ ) and $S c C B L 6$ (5'-TCTAGATGTGCAATGGTGGACTTTGT-3': XbaI and 5'-GGTAC CCGCATCCTCTACTTGAGAGTTGA-3': KpnI). The ScCIPK8 coding sequence was digested, purified, and cloned into the $p S P Y C E 35 S$ vector (Walter et al., 2004), generating the construct $p S P Y C E 35 S:: S c C I P K 8$, which was used to express the ScCIPK8 protein fused to the C-terminal fragment of yellow fluorescent protein (YFP). The ScCBL1, $S c C B L 3$, and ScCBL6 genes were digested, purified, and cloned into the pSPYNE35S vector to produce ScCBL proteins fused to the N-terminal fragment of YFP. Each BiFC construct was transformed individually into Agrobacterium tumefaciens by the freezethaw method (Holsters et al., 1978). A. tumefaciens that was transformed with the pSPYCE35S::ScCIPK8 construct was co-infiltrated in 3-week-old Arabidopsis leaves along with $A$. tumefaciens that was transformed with $S c C B L 1, S c C B L 3$, or $S c C B L 6$ that were cloned into $p S P Y N E 35 S$ according to Lee and Yang (2006). As a negative control, $A$. tumefaciens that was transformed with the $p S P Y C E 35 S$ empty vector was co-infiltrated in 3-week-old Arabidopsis leaves along with A. tumefaciens that was transformed with the pSPYNE35S empty vector. The fluorescence of the lower epidermis of Arabidopsis leaves were observed $16 \mathrm{~h}$ after infiltration on a Zeiss LSM780-NLO confocal laser scanning system. The YFP was detected by excitation with an argon laser $(488 \mathrm{~nm})$ and emission at 492 to $593 \mathrm{~nm}$. 


\section{RESULTS}

\section{Cloning and molecular characterization of ScCIPK8}

Previous studies showed that the SAS SCEQLB2019B08.g is induced by drought stress and is also related to sucrose content (Rocha et al., 2007; Felix et al., 2009). This SAS was first annotated from the SUCAST catalog as caneCIPK- 8 because it was the eighth sugarcane CIPK SAS to be annotated (Rocha et al., 2007). Here, we renamed the gene $S c C I P K 8$. The complete coding sequence of $S c C I P K 8$ was identified, and it comprises 1314 nucleotides encoding a putative protein of 437 amino acids (Table 1). The theoretical molecular mass and isoelectric point were estimated to be $50 \mathrm{kDa}$ and 6.82 , respectively. The deduced amino acid sequence is very similar to that of the kinase protein AtCIPK3 from Arabidopsis (76.3\% identity and 88.3\% amino acid similarity). Multiple alignments with several ScCIPK proteins from diverse plants were conducted (data not shown) and allowed us to produce a schematic view of the distinct putative domains of the ScCIPK8 protein (Figure 1). The N-terminal kinase domain of the ScCIPK8 protein is delimited by amino acids 1 and 265 and harbors an activation loop between amino acids 151 and 180 . The autoinhibitory C-terminal domain is delimited by amino acids 305 and 437. A junction domain that is delimited by amino acids 266 and 304 connects the $\mathrm{N}$-terminal and C-terminal domains of ScCIPK8. The NAF domain is located at the C-terminal regulatory domain, between amino acids 305 and 327. A putative protein phosphatase interaction (PPI) domain was also identified at the C-terminal region, located between amino acids 331 and 367.

\begin{tabular}{|c|c|c|c|c|c|}
\hline Name & SUCEST SAS ID. ${ }^{a}$ & $\mathrm{cDNA}^{\mathrm{b}}$ & Amino acid ${ }^{c}$ & $\mathrm{Mr}^{\mathrm{d}}$ & $\mathrm{pI}^{\mathrm{e}}$ \\
\hline ScCIPK8 & SCEQLB2019B08.g & 1314 & 437 & 50 & 6.82 \\
\hline ScCBL1 & SCRLLR1131D03.g & 645 & 214 & 24.5 & 4.69 \\
\hline ScCBL2 & SCEZRT2022G03.g & 678 & 225 & 25.9 & 4.8 \\
\hline ScCBL3 & SCJFRZ1007A06.g & 678 & 225 & 25.8 & 4.82 \\
\hline ScCBL6 & SCUTFL1058G06.g & 642 & 213 & 25.7 & 4.82 \\
\hline ScCBL9 & SCCCRZ2004D09.g & 1053 & 350 & 39.5 & 5.55 \\
\hline ScCBL10 & SCJFRZ2013C05.g & 798 & 265 & 30.1 & 4.87 \\
\hline
\end{tabular}

${ }^{a}$ SUCEST database SAS accession number. ${ }^{b} \mathrm{cDNA}$ length in bp. ${ }^{\mathrm{c} A m i n o}$ acid length of predicted protein. dTheoretical molecular mass (Mr) of the predicted protein calculated by ProParam Tool (http://web.expasy.org/

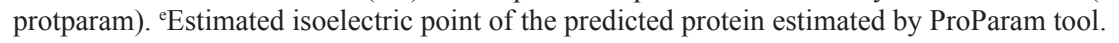

\section{Cloning and molecular characterization of $S c C B L s$}

Six SASs corresponding to putative $S c C B L$ genes were identified and named according to their similarities with Arabidopsis and rice $C B L$ genes: $S c C B L 1, S c C B L 2, S c C B L 3$, $S c C B L 6, S c C B L 9$, and $S c C B L 10$ (Table 1). Our results show that the sugarcane CBL protein family is quite conserved in size and structure, with identities and similarities in amino acid sequences ranging from 53 and $67.8 \%$ between ScCBL2 and ScCBL9 and 93.3 and $99.1 \%$ between ScCBL2 and ScCBL3. Similarly, high levels of conservation are observed in Arabidopsis and rice (Kolukisaoglu et al., 2004). The complete coding sequences of the distinct sugarcane $C B L$ genes vary from 642 ( $S C C B L 6$ ) to 1053 (ScCBL9) nucleotides (Table 1), and the predicted amino acid sequences vary from 213 (ScCBL6) to 350 (ScCBL9) amino acids. The 


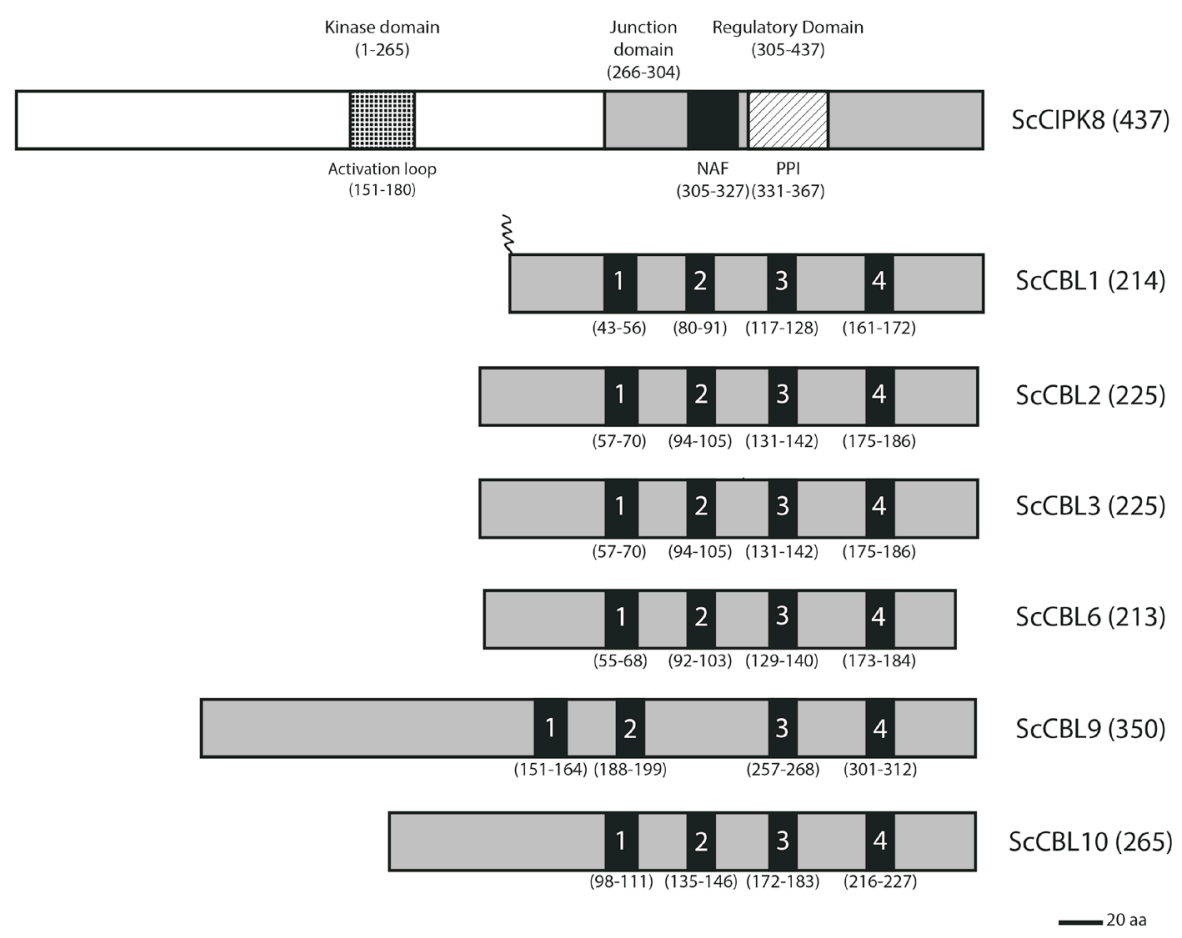

Figure 1. Schematic view of ScCIPK8 and the six ScCBLs predicted amino acid sequences. Start and ending points of the corresponding predicted domains are shown below each domain. The EF-hands in each ScCBL are indicated (numbers 1-4). The coil at the beginning of the N-terminus of ScCBL1 indicates the predicted myristoylation and palmitoylation site.

theoretical molecular mass ranges from 24.5 (ScCBL1) to $39.5 \mathrm{kDa}$ (ScCBL9), and the estimated isoelectric point ranges from 4.69 (ScCBL1) to 5.55 (ScCBL9) (Table 1). As observed with other CBL proteins from other plants, sugarcane CBLs have a conserved core comprised by their EF-hands and less conserved N- and C-terminal regions (Batistic and Kudla, 2004). In all ScCBLs that were identified, the first EF-hand consists of 14 amino acids, while the next three consist of 12 amino acids (Figure 1). In addition, the spacing regions connecting the different EF-hands have a constant number of amino acids. EF-hands 1 and 2 are spaced by 23 amino acids, EF-hands 2 and 3 are spaced by 25 amino acids, and EF-hands 3 and 4 are spaced by 32 amino acids. The only exception is the spacing region connecting EF-hand 2 and EF-hand 3 from ScCBL9, which has 57 amino acids and not the usual 25 amino acids. This region in ScCBL9 seems to have an insertion of 32 amino acids, making it different from all other CBLs described so far. Interestingly, ScCBL1 has a predicted myristoylation and palmitoylation site at its $\mathrm{N}$-terminus, which was supported by in silico analysis.

\section{ScCIPK8 protein-protein interaction network identification}

In order to verify if ScCIPK8 could interact with any of the ScCBLs that were identified, yeast two-hybrid assays were conducted. Among the six ScCBL proteins tested, the 
ScCIPK8 protein could interact with ScCBL1, ScCBL3, and ScCBL10 proteins in yeast cells (Figure 2A). The interactions that were identified in yeast two-hybrid assays were confirmed by BiFC assays in Arabidopsis leaf cells. Although it was not possible to observe the precise localization of these interactions, our data suggest that the interaction between ScCIPK 8 and ScCBL1 may occur either at the plasma membrane or at the cytoplasm (Figure 2B; ii), the interaction between ScCIPK8 and ScCBL3 may occur at the cytoplasm (Figure 2B; iii), and the interaction between ScCIPK8 and ScCBL6 may occur at a membrane compartment like the endoplasmic reticulum, tonoplast, or plasma membrane (Figure 2B; iv).

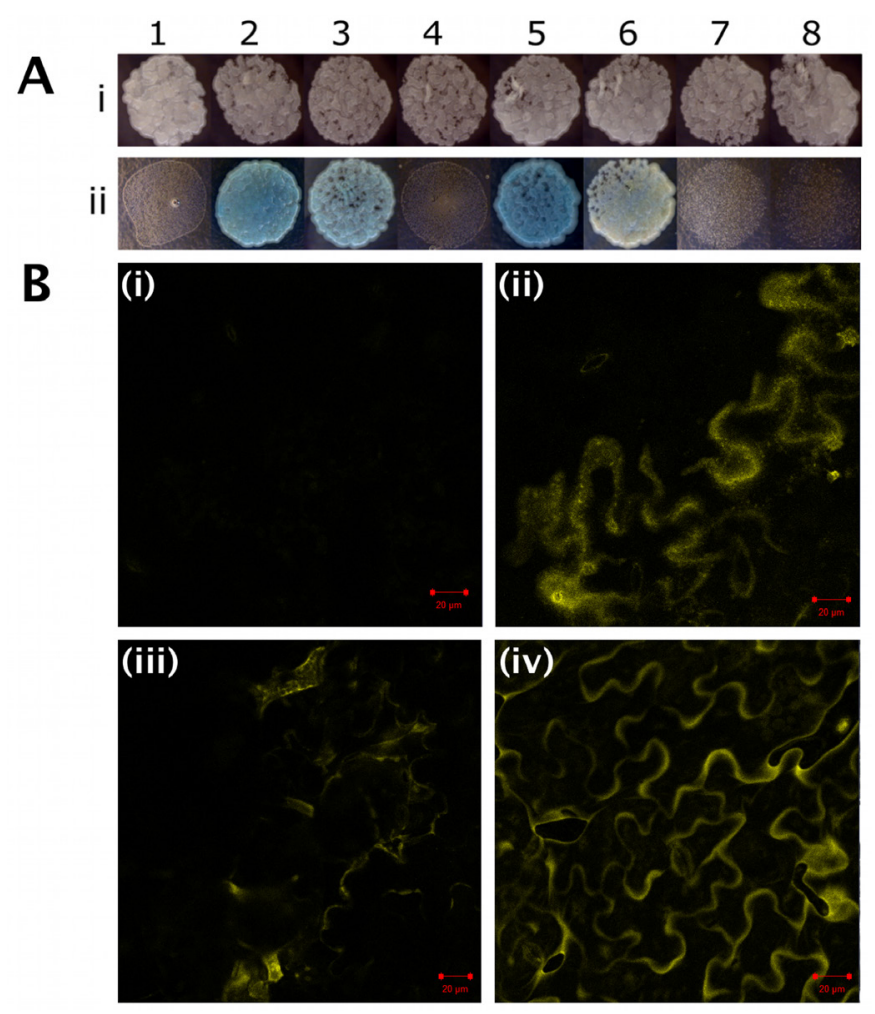

Figure 2. ScCIPK8 interaction with ScCBL proteins A. Yeast two-hybrid assays. i: SD medium lacking Leu and Trp amino acids; ii: SD medium lacking Leu, Trp, His, and Ade. 1, pGBKT7::53 + pGADT7::Lam (negative control). 2, pGBKT7::53 + pGADT7::T (positive control). 3, ScCBL1 + ScCIPK8. 4, ScCBL2 + ScCIPK8. 5, $S c C B L 3+S c C I P K 8.6, S c C B L 6+S c C I P K 8.7, S c C B L 9+S c C I P K 8.8, S c C B L 10+S c C I P K 8$. B. BiFC assays. Leaves of 3-week-old Arabidopsis thaliana were transiently transformed with Agrobacterium tumefaciens. i: pSPYCE + pSPYNE (negative control); ii: pSPYCE::SCCIPK8 + pSPYNE::ScCBL1; iii: pSPYCE::ScCIPK8 + pSPYNE::ScCBL3; iv: $p S P Y C e:: S c C I P K 8+p S P Y N E:: S c C B L 6$.

\section{DISCUSSION}

CIPKs and CBLs are usually involved in stress responses and sugar signaling in plants. In different stress conditions, CBLs can sense cellular calcium signals and activate CIPK proteins, which can phosphorylate downstream proteins. Although some CIPKs and 
CBLs have been described and functionally studied in other plants (Guo et al., 2001, 2002; Gong et al., 2002; Luan et al., 2002; Kolukisaoglu et al., 2004; Lee et al., 2005; Xiang et al., 2007; Tripathi et al., 2009), little is known about the proteins in sugarcane. It has been shown that the SCCIPK8 gene is induced by drought stress (Rocha et al., 2007) and that the expression levels are negatively correlated with sucrose content (Felix et al., 2009).

In order to better understand the role of ScCIPK8 in stress signaling and sugar responses, we cloned and characterized the ScCIPK8 gene and described its protein-protein interaction network with sugarcane CBL proteins. The ScCIPK8 ORF is 1314 bp and encodes a 437-amino acid sequence. Similar to other CIPK proteins, the deduced ScCIPK8 amino acid sequence has an $\mathrm{N}$-terminal kinase domain, a regulatory autoinhibitory C-terminal domain, which harbors the NAF domain and is responsible for CBL interactions, and a putative PPI domain. In Arabidopsis, a PPI domain that is present in the SOS2 protein (AtCIPK24) has been described as being responsible for the interaction with the protein phosphatase 2C ABI2 (Ohta et al., 2003). The PPI domain could act along with the NAF domain to fine-tune the ScCIPK8 activation state by modulating its phosphorylation/dephosphorylation in response to different environmental stimuli.

We identified and cloned six $S c C B L$ cDNAs. Sequence analysis of their deduced amino acid sequence showed that all of the identified ScCBLs have 4 EF-hands and that sugarcane CBL proteins constitute an evolutionary highly conserved family of proteins. It was shown that the ScCIPK8 protein could interact with the ScCBL1, ScCBL3, and ScCBL6 proteins but not with ScCBL2, ScCBL9, and ScCBL10 in yeast and in Arabidopsis leaf cells.

The interaction between ScCIPK8 and ScCBL1 may occur either at the plasma membrane or at the cytoplasm. This is in agreement with in silico analysis indicating that ScCBL1 is probably myristoylated and palmitoylated. ScCBL1 shows $77.5 \%$ identity and $91.1 \%$ amino acid similarity with the Arabidopsis AtCBL1 protein, which is known to be myristoylated and then palmitoylated at its N-terminus (Batistic et al., 2008). This dual lipid modification at AtCBL1 is responsible for directing the AtCIPK23-AtCBL1 complex to the plasma membrane, where AtCIPK23 can phosphorylate and activate the potassium $\left(\mathrm{K}^{+}\right)$channel protein AKT1 (Lee et al., 2007).

In vivo assays of the interaction between ScCIPK8 and ScCBL3, which is very similar to Arabidopsis AtCBL3 (86.6 and 94.2\% identity and amino acid similarity, respectively), suggest that these proteins interact at the cytoplasm. This pattern differs from the subcellular localization of the Arabidopsis AtCBL3 protein, which is localized at the tonoplast (Batistic et al., 2010). On the other hand, the interaction between ScCIPK8 and ScCBL6, which is also very similar to Arabidopsis AtCBL3 (82.4 and 92.8\% identity and amino acid similarity, respectively), may occur at a membrane compartment like the endoplasmic reticulum, tonoplast, or plasma membrane.

In summary, the identification of the $\mathrm{ScCIPK} 8 / \mathrm{ScCBL}$ protein-protein interaction network indicates that ScCIPK8 activation in sugarcane involves at least three CBL proteins. Considering that ScCIPK8 is induced by drought stress and has a negative relationship with sucrose accumulation, our data indicate that these CBLs can also play a role in regulating these responses in sugarcane.

\section{ACKNOWLEDGMENTS}

We acknowledge Conselho Nacional de Desenvolvimento Científico e Tecnológico (CNPq) for the doctoral scholarship for T.F. Farani. Research supported by grants \#2008/57986 (São Paulo Research Foundation - FAPESP) and \#574002/2008-1 (CNPq). 


\section{REFERENCES}

Albrecht V, Ritz O, Linder S, Harter K, et al. (2001). The NAF domain defines a novel protein-protein interaction module conserved in $\mathrm{Ca}^{2+}$-regulated kinases. EMBO J. 20: 1051-1063.

Batistic O and Kudla J (2004). Integration and channeling of calcium signaling through the CBL calcium sensor/CIPK protein kinase network. Planta 219: 915-924.

Batistic O, Sorek N, Schultke S, Yalovsky S, et al. (2008). Dual fatty acyl modification determines the localization and plasma membrane targeting of CBL/CIPK Ca ${ }^{2+}$ signaling complexes in Arabidopsis. Plant Cell 20: 1346-1362.

Batistic O, Waadt R, Steinhorst L, Held K, et al. (2010). CBL-mediated targeting of CIPKs facilitates the decoding of calcium signals emanating from distinct cellular stores. Plant J. 61:211-222.

Bologna G, Yvon C, Duvaud S and Veuthey AL (2004). N-Terminal myristoylation predictions by ensembles of neural networks. Proteomics 4: 1626-1632.

DeFalco TA, Bender KW and Snedden WA (2010). Breaking the code: $\mathrm{Ca}^{2+}$ sensors in plant signalling. Biochem. J. 425: $27-40$.

Felix JDM, Papini-Terzi FS, Rocha FR, Vêncio RZN, et al. (2009). Expression profile of signal transduction components in a sugarcane population segregating for sugar content. Trop. Plant Biol. 2: 98-109.

Gasteiger E, Hoogland C, Gattiker A, Duvaud S, et al. (2005). Protein Identification and Analysis Tools on the ExPASy Server. In: The Proteomics Protocols Handbook (Walker JM, ed.). Humana Press, New York, 571-607.

Gong D, Gong Z, Guo Y, Chen X, et al. (2002). Biochemical and functional characterization of PKS11, a novel Arabidopsis protein kinase. J. Biol. Chem. 277: 28340-28350.

Goujon M, McWilliam H, Li W, Valentin F, et al. (2010). A new bioinformatics analysis tools framework at EMBL-EBI. Nucleic Acids Res. 38: W695-W699.

Guo Y, Halfter U, Ishitani M and Zhu JK (2001). Molecular characterization of functional domains in the protein kinase SOS2 that is required for plant salt tolerance. Plant Cell 13: 1383-1400.

Guo Y, Xiong L, Song CP, Gong D, et al. (2002). A calcium sensor and its interacting protein kinase are global regulators of abscisic acid signaling in Arabidopsis. Dev. Cell 3: 233-244.

Hassan A, Okuta T, Kato M, Hatsugai N, et al. (2012). Alternaric acid stimulates phosphorylation of His-tagged RiCDPK2, a calcium-dependent protein kinase in potato plants. Genet. Mol. Res. 11: 2381-2389.

Holsters M, de Waele D, Depicker A, Messens E, et al. (1978). Transfection and transformation of Agrobacterium tumefaciens. Mol. Gen. Genet. 163: 181-187.

Kolukisaoglu U, Weinl S, Blazevic D, Batistic O, et al. (2004). Calcium sensors and their interacting protein kinases: genomics of the Arabidopsis and rice CBL-CIPK signaling networks. Plant Physiol. 134: 43-58.

Kudla J, Xu Q, Harter K, Gruissem W, et al. (1999). Genes for calcineurin B-like proteins in Arabidopsis are differentially regulated by stress signals. Proc. Natl. Acad. Sci. U. S. A. 96: 4718-4723.

Larkin MA, Blackshields G, Brown NP, Chenna R, et al. (2007). Clustal W and Clustal X version 2.0. Bioinformatics 23: 2947-2948.

Lee EJ, Iai H, Sano H and Koizumi N (2005). Sugar responsible and tissue specific expression of a gene encoding AtCIPK14, an Arabidopsis CBL-interacting protein kinase. Biosci. Biotechnol. Biochem. 69: 242-245.

Lee MW and Yang Y (2006). Transient expression assay by agroinfiltration of leaves. Methods Mol. Biol. 323: 225-229.

Lee SC, Lan WZ, Kim BG, Li L, et al. (2007). A protein phosphorylation/dephosphorylation network regulates a plant potassium channel. Proc. Natl. Acad. Sci. U. S. A. 104: 15959-15964.

Luan S, Kudla J, Rodriguez-Concepcion M, Yalovsky S, et al. (2002). Calmodulins and calcineurin B-like proteins: calcium sensors for specific signal response coupling in plants. Plant Cell 14 (Suppl): S389-S400.

Ohta M, Guo Y, Halfter U and Zhu JK (2003). A novel domain in the protein kinase SOS2 mediates interaction with the protein phosphatase 2C ABI2. Proc. Natl. Acad. Sci. U. S. A. 100: 11771-11776.

Ren J, Wen L, Gao X, Jin C, et al. (2008). CSS-Palm 2.0: an updated software for palmitoylation sites prediction. Protein Eng. Des. Sel. 21: 639-644.

Rocha FR, Papini-Terzi FS, Nishiyama MY Jr, Vêncio RZ, et al. (2007). Signal transduction-related responses to phytohormones and environmental challenges in sugarcane. BMC Genomics 8: 71.

Shi J, Kim KN, Ritz O, Albrecht V, et al. (1999). Novel protein kinases associated with calcineurin B-like calcium sensors in Arabidopsis. Plant Cell 11: 2393-2405.

Tripathi V, Parasuraman B, Laxmi A and Chattopadhyay D (2009). CIPK6, a CBL-interacting protein kinase is required for development and salt tolerance in plants. Plant J. 58: 778-790.

Walter M, Chaban C, Schutze K, Batistic O, et al. (2004). Visualization of protein interactions in living plant cells using bimolecular fluorescence complementation. Plant J. 40: 428-438.

Webb AAR, McAinsh MR, Taylor JE and Hetherington AM (1996). Calcium Ions as Intracellular Second Messengers in Higher Plants. In: Advances in Botanical Research (Callow JA, ed.). Academic Press, New York, 45-96.

Xiang Y, Huang Y and Xiong L (2007). Characterization of stress-responsive CIPK genes in rice for stress tolerance improvement. Plant Physiol. 144: 1416-1428. 